\title{
Exclusive breastfeeding rate and related factors among mothers within maternal health WeChat groups in Jiaxing, Zhejiang province, China: A cross-sectional survey
}

Chun Y. Fu Jiaxing University

Xue J. Tang Jiaxing University

Ling P. Pan

Jiaxing University

Hai Y. Jin Jiaxing University

Juan F. Yao Jiaxing University

Li-Zhong Wang ( $\nabla_{\text {jxlzw@sina.com ) }}$ jiaxing maternity and children health care hospital https://orcid.org/0000-0002-2654-5178

\section{Research}

Keywords: Cross-sectional study, Exclusive breastfeeding, Maternal health care, WeChat application

Posted Date: November 9th, 2021

DOl: https://doi.org/10.21203/rs.3.rs-1046017/v1

License: (c) (i) This work is licensed under a Creative Commons Attribution 4.0 International License. Read Full License 


\section{Abstract \\ Background}

The benefits of exclusive breastfeeding (EBF) in the first six months are well recognized, but the EBF rate is still lower in China. WeChat, a most widely used communication application in China, is now used for maternal health education and management in Jiaxing, Zhejiang province, China. The aim of this study was to assess the EBF rate and its associated factors among the mothers within the maternal health WeChat groups.

\section{Methods}

This was a cross-sectional survey on healthy mothers with infants aged 7-12 months from seven maternal health WeChat groups in Jiaxing, China. Data including breastfeeding practice in the first six months, maternal sociodemographic and obstetric characteristics, hospitalization information, work related factors and reasons for non-EBF were collected using an online self-administered questionnaire in October 2021. A multivariable logistic regression analysis was performed to identify the factors independently associated with EBF in the first six months.

\section{Results}

A total of 822 mothers were included in this study. Among them, 586 mothers exclusively breastfed their infants until six months with a EBF rate of $71.3 \%$. Multivariable logistic regression analysis indicated that maternal age (adjusted odds ratio, AOR: $0.948,95 \% \mathrm{Cl}$ : 0.909-0.990) and perceived insufficient breast milk (AOR: $0.104,95 \% \mathrm{Cl}: 0.072-0.150$ ) were negatively associated with EBF, while EBF during hospitalization was positively associated with EBF within six months (AOR: 2.889, 95\% Cl: 2.014-4.143). The five of common reasons for non-EBF were no or insufficient breast milk (59.8\%), return to work (23.9\%), no flexible nursing breaks at work (18.2\%), infant crying or feeling tired or troubled with breastfeeding $(9.7 \%)$, and nipple and breast problems $(9.3 \%)$.

\section{Conclusion}

The EBF rate is higher among the mothers within maternal health WeChat groups. WeChat group model can be used to be a cost-effective and useful measure to promote EBF in China. However, perceived insufficient breast milk and work related factors are still the main barriers to EBF in this setting.

\section{Background}

A wide range of benefits of breastfeeding for both infants and mothers in the short and long term are well-known [1]. World Health Organization (WHO) recommends exclusively breastfeeding (EBF) of infants 
for the first six months after birth and continued breastfeeding with complementary foods until two years or beyond [2]. In 2012, the 56th World Health Assembly set as a target for 2025 to increase the EBF rate within six months up to at least $50 \%$ [3]. It is estimated that, if scaled up to a near-universal level, breastfeeding can prevent 823000 deaths in children under five years of age and 20000 breast cancer deaths every year [1]. In China, likewise, assuming an increase to $50 \%$ of EBF at six months could reduce the mortality of children under five years of age by $5 \%[4]$.

However, the EBF rates remain suboptimal in many countries around the world [1]. The global EBF rate for infants 0-6 months old is now about $44 \%$ [2]. In China, despite many years of efforts to improve breastfeeding, the EBF rates were $20.7 \%$ in 2013 [5] and 29.5\% in 2018 [6] as estimated by the nationally representative surveys; in the local studies, the reported EBF rates were $28.7 \%$ in central and western China in 2010 [7], 26.7\% in Hong Kong in 2013 [8], 27.34\% in Southwest China in 2017 [9], and 37.0\% in Nanning in 2019 [10]. Hence, the EBF rates in China are well lower than the global average level and the international target, which has become the main challenge for Chinese public health care. To dramatically increase EBF rates in China, it is necessary to continuously monitor the trend of EBF rates and explore more effective interventions to promote breastfeeding practice [6].

There are many factors that can affect breastfeeding, of which education and support provided by health workers play an important role in breastfeeding outcomes [8-13]. Jiaxing is a medium-sized city in Zhejiang province, a relatively developed area on the eastern coast of China. The city consists of five counties and two districts with a population of 5400868 and 37366 births in 2020. Based on a past sample survey, the EBF rate within six months in Jiaxing was only $13.2 \%$ in 2007 [14]. Due to the widespread use of WeChat, a free and popular online communication application in mainland China, we implemented a novel model of maternal health care for perinatal women by establishing dedicated WeChat groups in 2019. The WeChat groups support one-on-one texting, group chats, multimedia sharing and more. Pregnant women are invited to join the WeChat groups when they attend the antenatal outpatient clinics. At now, each county and district in Jiaxing has established its own maternal health WeChat group, each having about 200-500 women. A dedicated health worker is designated as the group manager who provides group education and individual counselling on breastfeeding for women in the group. We wanted to know if this education and management model as part of traditional maternal health care work could improve EBF rate. Therefore, we conducted this cross-sectional survey to assess the prevalence of EBF in the fist six months among the mothers who had joined in the maternal health WeChat groups in Jiaxing, China. The related factors potentially affecting EBF in this setting were also assessed.

\section{Methods}

\section{Study design}

This is a cross-sectional study which was conducted using an online self-administered questionnaire in October 2021 in Jiaxing, Zhejiang province, China. The inclusion criteria were healthy mothers with 
infants aged 7-12 months in our seven maternal health WeChat groups. The exclusion criteria were multiple gestations, premature delivery before 34 weeks, mastitis or prior breast surgery, major fetal congenital malformations, or any other problems that could affect breastfeeding. The study is reported according to the Strengthening the Reporting of Observational Studies in Epidemiology (STROBE) guidelines [15].

\section{Data collection}

The questionnaire was designed by the researchers based on previous studies $[6,11]$ to address the survey objectives. It comprised a total of 28 items by using dichotomous, multiple-choice or open-ended questions, including breastfeeding practice in the first six months, maternal sociodemographic characteristics, knowledge about the general breastfeeding recommendations by WHO (i.e. optimal infant breastfeeding should be initiated within the first hour of birth, EBF continue for six months and then appropriate complementary feeding should commence together with breastfeeding for at least two years) [2], obstetric information, breastfeeding support during hospitalization, and work related factors (Table 1). There were three options for the breastfeeding practice in the first six months (i.e. EBF, mixed feeding or no breastfeeding). EBF was defined as the infant only received breast milk without any additional food or drink, except for medicines, vitamins, and minerals within six months [1]. Participants who chose "mixed feeding" or "no breastfeeding" were grouped into non-EBF group and were asked to furtherly report the reasons for non-EBF (open-ended and can be multiple).

Table 1

Type and contents of questions in the self-administered questionnaire

\begin{tabular}{|ll}
\hline Type & Contents \\
\hline Dichotomous & $\begin{array}{l}\text { Parity, marital status, resident area, household register, knowledge about the } \\
\text { breastfeeding recommendations, pregnancy and postnatal complications, baby } \\
\text { gender, skin-to-skin contact, rooming-in, breastfeeding initiation after birth, EBF } \\
\text { during hospitalization, perception of insufficient breast milk, paid maternity leave, } \\
\text { breastfeeding room at workplace }\end{array}$
\end{tabular}

Multiple- $\quad$ Breastfeeding practice in the first six months, educational level, employment status, choice monthly family incomes, mode of delivery,

Open-ended Age, height, weight, birth weight, ethnic origin, gestational age, reasons for non EBF

EBF: exclusive breastfeeding.

The questionnaire was designed as simple as possible for participants to easily understand and make judgments. Prior to the formal release, the questionnaire was pretested among 10 unselected mothers to evaluate the appropriate wording and understandability. Then the questionnaire was online released in the seven WeChat groups and all eligible mothers were invited to this survey. The group managers were responsible for giving a full explanation to potential participants about the purpose of the survey, eligibility and exclusion criteria, and the voluntary and anonymity nature of the study including their right to not to participate in or to withdraw from the study at any time, but once they submitted the questionnaire meant they agreed to participate in the study. If agreed, participates were asked to 
complete and submit the questionnaire online on their mobile phones within two weeks. During data collection, the group managers answered any questions raised by the participants regarding the questionnaire contents.

\section{Ethical considerations}

This study was approved by the Local Research Ethics Committee of Jiaxing Maternity and Children Health Care Hospital (Approval No. 2021-F-61). It was an online voluntary and anonymous survey, participants were informed that completing and submitting the questionnaire meant they agreed to participate in the study. As such, the requirement for written informed consent was waived by the Ethics Committee.

\section{Data analysis}

The primary outcome was the self-reported EBF rate in the fist six months postpartum. Continuous variables were presented as median (range), categorical variables were presented as number and percentage. Normal distribution for continuous variables was checked using the Shapiro-Wilk test and visual plot inspection. As the continuous variables were not normally distributed, univariate analysis was performed using Mann-Whitney Utest, or the $x^{2}$ test and Fisher's exact test to compare the differences between mothers with and without EBF. A multivariable logistic regression model was used to determine the different independent variables associated with EBF by the adjusted odds ratio (AOR) and $95 \%$ confidence interval $(\mathrm{Cl})$. The independent variables that entered into the regression model were those of being significant in univariate analysis $(P<0.10)$ or of possibly influencing EBF indicated by previous studies. Multicollinearity was assessed using the tolerance and variance inflation factor, with tolerance > 0.10 and variance inflation factor $<10.0$ were considered acceptable. We did not perform a formal sample size calculation, but used a convenience sampling method. Data were analyzed using SPSS version 19.0 for Windows (IBM Corp., Armonk, NY, USA). A two-sided $P<0.05$ was considered as statistically significant.

\section{Results}

\section{Sample characteristics}

Based on the estimates of the groups managers, there were about 1,140 eligible mothers in the seven WeChat groups at the survey time. A total of 857 mothers online completed and submitted the questionnaire (response rate was $75.2 \%$ ). Thirty-five mothers were excluded due to multiple gestations $(n=27)$, premature delivery before 34 weeks $(n=5)$, postpartum severe hemorrhage $(n=2)$ or infections $(n=1)$, leaving 822 mothers included in this study. There were no significant differences in basal characteristics between excluded and included mothers (data not shown). All submitted questionnaires were complete.

The median age of participants was 29.6 (range 17-47) years. All participants were of Han ethnic and received a junior high school education or above (i.e., all had completed at least the nine-year compulsory 
education). The majority of surveyed mothers were married (98.1\%), employed full-time or part-time (86.5 $\%$ ) with a monthly family income of more than $5000 \mathrm{RMB}(91.1 \%)$, and aware of the general breastfeeding recommendations (86.1\%). All mothers gave birth at seven public hospitals located in Jiaxing, of which five had Baby-Friendly Hospital Initiative (BFHI) certificates. With respect to obstetric and hospitalization information, $89.9 \%$ of mothers were term (> 37 weeks), $67.3 \%$ were primiparous, $65.3 \%$ had a vaginal delivery, $92.5 \%$ were room-in, $88.6 \%$ had skin-to-skin contact with their infants immediately after birth, $67.2 \%$ commenced breastfeeding within the first hour of birth and $83.3 \%$ practiced EBF during hospitalization. In addition, of the full-time employed mothers $(n=480)$, the majority $(87.5 \%)$ returned to work after a paid maternity leave of 128 days for vaginal delivery and 143 days for cesarean delivery, but $72.7 \%$ reported that there was no independent breastfeeding room at their workplaces.

\section{EBF rate and associated factors}

Overall, 586 mothers reported that they exclusively breastfed their infants until six months, with a EBF rate of $71.3 \%$ (95\% $\mathrm{Cl}: 68.2-74.4 \%)$. Univariate analysis showed significant differences in maternal age $(P=0.001)$, body mass index (BMI) $(P=0.015)$, employment status $(P=0.003)$, mode of delivery $(P=0.014)$, breastfeeding initiation within the first hour $(P=0.017)$, EBF during hospitalization $(P<0.001)$, and perception of insufficient breast milk $(P<0.001)$ between mothers with and without EBF (Table 2). After adjustment for other variables, multivariable regression analysis indicated that only maternal age (AOR: 0.948, 95\% Cl: 0.909-0.990) and perceived insufficient breast milk (AOR: 0.104, 95\% Cl: 0.072-0.150) were negatively associated with EBF, while EBF during hospitalization (AOR: 2.889, 95\% Cl: 2.014-4.143) was positively associated with EBF (Table 3 ). 
Table 2

Related Variables in mothers with and without EBF

\begin{tabular}{|c|c|c|c|}
\hline Variables & EBF $(n=586)$ & Non-EBF $(n=236)$ & $P$ \\
\hline Maternal age, years & $29(17-43)$ & $30(21-47)$ & 0.001 \\
\hline $\mathrm{BMI}, \mathrm{kg} \cdot \mathrm{m}^{-2}$ & $21.5(15.6-38.5)$ & $22.1(15.8-35.2)$ & 0.015 \\
\hline Household registration & $411(70.1)$ & $176(74.6)$ & 0.202 \\
\hline Local & $175(29.9)$ & $60(25.4)$ & \\
\hline \multicolumn{4}{|l|}{ Non-local } \\
\hline Resident area & $348(59.4)$ & $147(62.3)$ & 0.442 \\
\hline Urban & $238(40.6)$ & $89(37.7)$ & \\
\hline \multicolumn{4}{|l|}{ Rural } \\
\hline Marital status & $572(97.6)$ & $234(99.2)$ & 0.174 \\
\hline Married & $14(2.4)$ & $2(0.8)$ & \\
\hline \multicolumn{4}{|l|}{ Unmarried } \\
\hline Educational level & $87(14.8)$ & $29(12.3)$ & 0.374 \\
\hline Junior high school or below & $101(17.2)$ & $35(14.8)$ & \\
\hline High school & $398(67.9)$ & $172(72.9)$ & \\
\hline \multicolumn{4}{|l|}{ College or above } \\
\hline Employment status & $83(14.2)$ & $28(11.9)$ & 0.003 \\
\hline Unemployed & $182(31.1)$ & $49(20.8)$ & \\
\hline Part-time & $321(54.8)$ & $159(67.4)$ & \\
\hline \multicolumn{4}{|l|}{ Full-time } \\
\hline Monthly family incomes, RMB & $49(8.4)$ & $24(10.2)$ & 0.057 \\
\hline$<5000$ & $244(41.6)$ & $97(41.1)$ & \\
\hline $5000-10000$ & $208(35.5)$ & $66(28)$ & \\
\hline 10 001-19 999 & $85(14.5)$ & $49(20.8)$ & \\
\hline$\geq 20000$ & & & \\
\hline
\end{tabular}

Data are presented as median (range) or number (percentage). EBF: exclusively breastfeeding, BMI: body mass index.

${ }^{a}$ From the full-time employed mothers ( $n=321$ in EBF, $n=159$ in non-EBF). 


\begin{tabular}{|c|c|c|c|}
\hline Variables & EBF $(n=586)$ & Non-EBF $(n=236)$ & $P$ \\
\hline Gestational age & $61(10.4)$ & $22(9.3)$ & 0.640 \\
\hline 34- 37 weeks & $525(89.6)$ & $214(90.7)$ & \\
\hline \multicolumn{4}{|l|}{ > 37 weeks } \\
\hline Parity & $399(68.1)$ & $154(65.3)$ & 0.433 \\
\hline Primiparas & $187(31.9)$ & $82(34.7)$ & \\
\hline \multicolumn{4}{|l|}{ Multiparas } \\
\hline Pregnancy complications & $46(7.8)$ & $21(8.9)$ & 0.619 \\
\hline Yes & $540(92.2)$ & $215(91.1)$ & \\
\hline \multicolumn{4}{|l|}{ No } \\
\hline Postnatal complications & $9(1.5)$ & $5(2.1)$ & 0.558 \\
\hline Yes & $577(98.5)$ & 231 (97.9) & \\
\hline \multicolumn{4}{|l|}{ No } \\
\hline Mode of delivery & $398(67.9)$ & $139(58.9)$ & 0.014 \\
\hline Vaginal delivery & $188(32.1)$ & $97(41.1)$ & \\
\hline \multicolumn{4}{|l|}{ Cesarean delivery } \\
\hline Birth weight, g & $21(3.6)$ & $13(5.5)$ & 0.210 \\
\hline$\leq 2500$ & $565(96.4)$ & $223(94.5)$ & \\
\hline \multicolumn{4}{|l|}{$>2500$} \\
\hline Baby sex & 309 (52.7) & $122(54.7)$ & 0.788 \\
\hline Male & $277(47.3)$ & $114(48.3)$ & \\
\hline \multicolumn{4}{|l|}{ Female } \\
\hline Rooming-in & $544(92.8)$ & $216(91.5)$ & 0.521 \\
\hline Yes & $42(7.2)$ & $20(8.5)$ & \\
\hline No & & & \\
\hline
\end{tabular}

Data are presented as median (range) or number (percentage). EBF: exclusively breastfeeding, BMI: body mass index.

${ }^{a}$ From the full-time employed mothers ( $n=321$ in EBF, $n=159$ in non-EBF). 


\begin{tabular}{|c|c|c|c|}
\hline Variables & EBF $(n=586)$ & Non-EBF $(n=236)$ & $P$ \\
\hline Breastfeeding initiation after birth & $408(69.6)$ & $144(61.0)$ & 0.017 \\
\hline$\leq 1 \mathrm{~h}$ & $178(30.4)$ & $92(39.0)$ & \\
\hline \multicolumn{4}{|l|}{$>1 \mathrm{~h}$} \\
\hline EBF during hospitalization & $540(92.2)$ & $145(61.4)$ & $<0.001$ \\
\hline Yes & $46(7.8)$ & $91(38.6)$ & \\
\hline \multicolumn{4}{|l|}{ No } \\
\hline Skin-to-skin contact after birth & $527(89.9)$ & $201(85.2)$ & 0.052 \\
\hline Yes & $59(10.1)$ & $35(14.8)$ & \\
\hline \multicolumn{4}{|l|}{ No } \\
\hline Knowledge about breastfeeding recommendations & $511(87.2)$ & $197(83.5)$ & 0.162 \\
\hline Yes & $75(12.8)$ & $39(16.5)$ & \\
\hline \multicolumn{4}{|l|}{ No } \\
\hline Perception of insufficient breast milk & $151(25.8)$ & $182(77.1)$ & $<0.001$ \\
\hline Yes & $435(74.2)$ & $54(22.9)$ & \\
\hline \multicolumn{4}{|l|}{ No } \\
\hline Breastfeeding room at workplace ${ }^{a}$ & $92(28.7)$ & $39(24.5)$ & 0.339 \\
\hline Yes & $229(71.3)$ & $120(75.5)$ & \\
\hline \multicolumn{4}{|l|}{ No } \\
\hline \multicolumn{4}{|c|}{$\begin{array}{l}\text { Data are presented as median (range) or number (percentage). EBF: exclusively breastfeeding, BMI: } \\
\text { body mass index. }\end{array}$} \\
\hline
\end{tabular}

Table 3

Multivariable logistic regression of the associated factors with EBF

\begin{tabular}{|lccc|}
\hline & AOR & $95 \% \mathrm{Cl}$ & $\boldsymbol{P}$ \\
\hline Maternal age & 0.948 & $0.909-0.990$ & 0.015 \\
\hline EBF during hospitalization & 2.889 & $2.014-4.143$ & $<0.001$ \\
\hline Perceived insufficient breast milk & 0.104 & $0.072-0.150$ & $<0.001$ \\
\hline EBF: exclusively breastfeeding; AOR: adjusted odds ratio; Cl: confidence interval. \\
\hline
\end{tabular}




\section{Reasons for non-EBF}

In 236 mothers who failed to EBF in the fist six months, the top five reasons given for non-EBF were no or insufficient breast milk (59.8\%), inability to breastfeed their infants as needed after return to work (23.9\%), no flexible nursing breaks at work (18.2\%), infant crying or feeling tired or troubled with breastfeeding $(9.7 \%)$, and nipple and breast problems (9.3\%), respectively. Other reasons included the concern that breast milk alone was not sufficient for infant's nutritional needs (7.2\%), infant's weight below the standard (6.8\%), no breastfeeding room or refrigerator for expressing or storing breast milk at workplace (6.8\%), perceived inconveniences or discomfort of breastfeeding in public (4.2\%), maternal illness (2.5\%) and pain or discomfort (1.7\%).

\section{Discussion}

The current study reveals that the EBF rate in the first six months among the mothers within the maternal health WeChat groups in Jiaxing, China, is $71.3 \%$, which is considerably higher compared to the rates reported by both national and local surveys in China [5-10]. Although there are other factors for this higher EBF rate, we tend to think that the implementation of WeChat group model as a supplement to traditional maternal health care is a main contributing factor.

Health workers have played an important role in breastfeeding initiation and continuation [8-12]. In the traditional model of maternal health care, women are required to attend antenatal and postnatal clinics or classes regularly, and health workers are needed to follow up the mothers by telephone calls or home visits after their hospital discharge. This could decrease the compliance of women and increase the workload of health workers, which in turn diminishes the effectiveness of maternal health care. Considering that WeChat is universally used by almost all women of childbearing age in our area, we adopt the establishment of maternal health WeChat groups as part of routine maternal health care. The advantages of WeChat group include its convenience and timeliness. In this way, health workers can provide regular breastfeeding education for all mothers and individual counseling for those who are experiencing feeding problems. The higher awareness rate $(86.1 \%)$ of breastfeeding recommendations in this study also reflects the effectiveness of this model of health education. Moreover, the mothers within the group can share their breastfeeding experiences with each other, thereby enhancing their breastfeeding confidence. In addition, this model also provides convenience for health workers to make investigations and gather information on some issues if needed.

We acknowledge, of course, that other factors can also contribute to this higher EBF rate in this study. In particular, most mothers gave birth at the BFHI certificate hospitals which adopted the "Ten Steps to Successful Breastfeeding" launched by WHO [16]. Therefore, it is not surprising that a higher proportion of mothers, even for those with cesarean delivery, had skin-to-skin contact immediately after birth, rooming-in with their infants, breastfeeding initiation within the first hour and EBF during hospitalization in this survey. These interventions have been shown to be strong contributors to establishment and continuation of breastfeeding [11, 17-20]. 
In line with previous studies [8, 9, 21-23], we found that there were significant differences in maternal age, BMI, employment status, mode of delivery, breastfeeding initiation, EBF during hospitalization and perception of insufficient breast milk between mothers with and without EBF base on the univariate analysis. However, multivariable regression analysis showed that only maternal age, EBF during hospitalization and perception of insufficient breast milk were associated with EBF. Especially, perceived insufficient breast milk significantly decreased, while EBF during hospitalization significantly increased the possibility of EBF within six months. Regarding the association between maternal age and breastfeeding, data in the literatures are conflicting. A number of studies observed that older mothers were associated with lower EBF compared to younger ones [6, 9]; in contrast, other studies showed that older mothers were more likely to have a positive attitude toward breastfeeding and to practice EBF than younger ones [22, 24]. In this study, maternal age was found to be negatively but marginally associated with EBF.

The lack of associations of other variables with EBF within six months may be due to the fact that the majority of surveyed mothers were employed and had breastfeeding initiation within the first hour. As for mode of delivery, previous studies have suggested that women with cesarean delivery were less likely to EBF than those with vaginal delivery $[6,17]$. Delayed onset of lactation, disrupted mother-infant interaction, inhibited infant suckling and poor pain relief may mediate the effects of caesarean delivery on breastfeeding [25]. In the current study, although caesarean delivery was less common in EBF mothers than in non-EBF mothers, it was not associated with EBF after adjustment for other confounding variables. This result is consistent with the study by Ruan et al [9], and suggests that if the mothers receive adequate breastfeeding support, especially during hospitalization, caesarean delivery is not necessarily a barrier to EBF. This may be especially important considering that a relatively higher caesarean delivery rate in China [26]. In addition, we did not found the difference in mothers' educational level, monthly family incomes, skin-to-skin contact, and room-in between mothers with and without EBF, those have been identified as the factors related to $\operatorname{EBF}$ in previous studies $[5,6,12,20]$. Again, sample characteristics and hospital practices may explain the discrepancy between this study and other studies.

With regard to the reasons for non-EBF, no or insufficient breast milk is the foremost one reported by the non-EBF mothers. This is consistent with the multivariable regression analysis and with other studies [6, $9,10]$. However, in fact, only few mothers have physiological insufficient milk supply and most mothers can produce enough breastmilk to meet their infant's demand [27]. As such, this result may imply the inadequate education and guidance provided by health workers on this issue. The next main reasons are those work related factors, including inability to breastfeed their infants as needed after return to work and lack of flexible breaks at work. Notably, although fewer mothers $(6.8 \%)$ stated no breastfeeding room or refrigerator at workplaces as the reason for non-EBF, $72.7 \%$ of the employed mothers reported there was no breastfeeding room at their workplaces. Other reasons for non-EBF are various, including infant crying or mother feeling tired or troubled with breastfeeding, nipple and breast problems, the concern about breast milk alone being not sufficient for infant's needs, and perceived inconveniences or discomfort of breastfeeding in public. Fortunately, most reasons listed above can be amended through education and intervention. For example, health workers can guide mothers how to tell the difference 
between physiological and perceived insufficient breast milk, prepare mothers for tiredness and fatigue, improve mothers' ability to soothe their infants, and eliminate their concern about insufficient breast milk nutrition. Returning to work before six months is still the common reason of early weaning breastfeeding for working mothers $[9,11,13]$. Thus, breastfeeding-friendly work policies and environments are needed for improving EBF among those mothers. For example, a relatively long maternity leave can extend breastfeeding duration for working mothers $[11,21,28]$. Hence, government may consider a longer paid maternity leave, guarantee frequent and flexible breaks at work, and encourage the provision of an independent breastfeeding room with a refrigerator at the workplaces. In Jiaxing city, women can now have a paid maternity leave of 128 days for vaginal delivery and 143 days for cesarean delivery.

\section{Limitations}

This study has some limitations. First, due to the nature of cross-sectional design, we cannot establish causal relationships between EBF and associated factors. Second, we used a convenience sampling method and only enrolled the mothers from our maternal health WeChat groups. It is feasible that women who have joined in the WeChat groups are more inclined to breastfeed. Further investigation that includes the whole obstetric population in the same city is warranted to confirm the effect of WeChat group on EBF. Third, the response rate was estimated to be about $75.3 \%$. It is possible that mothers who did not practice EBF were less willing to respond than those who did, which may also lead to overestimation of the EBF rate. Fourth, because the mothers completed the questionnaire six months after their delivery, the recall bias could not be avoided. In addition, the self-report nature of the study may also cause reporting bias. Finally, the questionnaire was designed to be relatively simple in order to increase the participation rate. There were some important factors that failed to be measured, such as mothers' intention and attitude to breastfeeding, the time when mothers introduce complementary foods and wean breastfeeding, supports of husbands and families, etc. These variables have previously been reported as the factors affecting $\operatorname{EBF}[7,11,28]$ and may provide more information for future breastfeeding education and interventions.

\section{Conclusions}

In summary, this study shows a higher EBF rate (71.3\%) in the first six months among the mothers within maternal health WeChat groups in Jiaxing, China. The higher EBF rate may attribute to multiple factors, but we believe that WeChat group model as part of maternal health education and management may have played an important role, as compared to those reported at the national and local levels in China. As a cost-effective and useful measure, WeChat group model can be considered to incorporate into the current breastfeeding promotion interventions in China. On the other hand, our study highlights that there is room for improvement of EBF even in this setting. The perceived insufficient breast milk and work related factors are still the main barriers to EBF in our study setting; therefor, future health care should provide more adequate breastfeeding education, especially relate to insufficient breast milk, and promote the provision of a breastfeeding-friendly work environment for working mothers. 


\section{Abbreviations}

AOR

Adjusted Odds Ratio

$\mathrm{Cl}$

Confidence Interval

EBF

Exclusively Breastfeeding

WHO

World Health Organization

\section{Declarations}

\section{Acknowledgements}

Not applicable

\section{Authors' contributions}

C.Y.F. and X.J.T. formulated the concept and design of the research. C.Y.F, L.P.P., H.Y.J. and J.F.Y. carried out the survey. C.Y. F. and L.Z.W. collected and analysed the data and completed the first draft. All authors contributed to revising the draft and approved the final manuscript.

\section{Funding}

Not applicable

\section{Availability of data and materials}

The datasets used and/or analysed during the current study are available from the corresponding author on reasonable request.

\section{Ethics approval and consent to participate}

This study was approved by the Local Research Ethics Committee of Jiaxing Maternity and Children Health Care Hospital (Approval No. 2021-F-61). As it was an online survey and participants were informed about the voluntary nature of the survey, the requirement for written informed consent was waived by the Ethics Committee.

\section{Consent for publication}

Not applicable

\section{Competing interests}


The authors declare that they have no competing interests

\section{Author details}

${ }^{1}$ Department of Maternal Health, Jiaxing Maternity and Children Health Care Hospital, Affiliated Women and Children Hospital, Jiaxing University, Jiaxing, Zhejiang Province, China. ${ }^{2}$ Department of Maternal Health, Jiaxing Xiuzhou Maternity and Children Health Care Hospital, Jiaxing, Zhejiang Province, China.

${ }^{3}$ Department of Public Health, Jiashan Maternity and Children Health Care Hospital, Jiaxing, Zhejiang Province, China. ${ }^{4}$ Department of Maternal Health, Tongxian Maternity and Children Health Care Hospital, Jiaxing, Zhejiang Province, China. ${ }^{5}$ Department of Anesthesiology, Jiaxing Maternity and Children Health Care Hospital, Affiliated Women and Children Hospital, Jiaxing University, Jiaxing, Zhejiang Province, China

\section{References}

1. Victora CG, Bahl R, Barros AJ, França GV, Horton S, Krasevec J, et al; Lancet Breastfeeding Series Group. Breastfeeding in the 21st century: epidemiology, mechanisms, and lifelong effect. Lancet. 2016; 387: 475-90. https://doi.org/10.1016/S0140-6736(15)01024-7.

2. World Health Organization. Infant and young child feeding. World Health Organization. 9 June 2021. Available from: https://www.who.int/news-room/fact-sheets/detail/infant-and-young-child-feeding. Accessed 22 October 2021.

3. World Health Organization. Global nutrition targets 2025: policy brief series. 20 December 2014. Available from: https://www.who.int/publications/i/item/WHO-NMH-NHD-14.2. Accessed 22 October 2021.

4. Zhenyu Yang, Jianqiang Lai, Dongmei Yu, Yifan Duan, Xuehong Pang, Shan Jiang, et al. Breastfeeding rates in China: a cross-sectional survey and estimate of benefits of improvement. The Lancet. 2016; 388 (S1): S47. https://doi.org/10.1016/S0140-6736(16)31974-2.

5. Duan Y, Yang Z, Lai J, Yu D, Chang S, Pang X, et al. Exclusive breastfeeding rate and complementary feeding indicators in China: A national representative survey in 2013. Nutrients. 2018;10: 249. https://doi.org/10.3390/nu10020249.

6. Shi H, Yang Y, Yin X, Li J, Fang J, Wang X. Determinants of exclusive breastfeeding for the first six months in China: A cross-sectional study. Int Breastfeed J. 2021;16: 40. https://doi.org/10.1186/s13006-021-00388-y.

7. Guo S, Fu X, Scherpbier RW, Wang Y, Zhou H, Wang X, et al. Breastfeeding rates in central and western China in 2010: implications for child and population health. Bull World Health Organ. 2013;91: 322-31. https://doi.org/10.2471/BLT.12.111310.

8. Wang W, Lau Y, Chow A, Chan KS. Breast-feeding intention, initiation and duration among Hong Kong Chinese women: a prospective longitudinal study. Midwifery. 2014; 30: 678-87. https://doi.org/10.1016/j.midw.2013.07.015. 
9. Ruan Y, Zhang Q, Li J, Wan R, Bai J, Wang W, et al. Factors associated with exclusive breast-feeding: A cross-sectional survey in Kaiyuan, Yunnan, Southwest China. PLoS One. 2019; 14: e0223251. https://doi.org/10.1371/journal.pone.0223251.

10. Li J, Zhao C, Wang Y, Wang YP, Chen CY, Huang Y, et al. Factors associated with exclusive breastfeeding practice among mothers in nine community health centres in Nanning city, China: a cross-sectional study. Int Breastfeed J. 2021;16: 71. https://doi.org/10.1186/s13006-021-00416-x.

11. Rollins NC, Bhandari N, Hajeebhoy N, Horton S, Lutter CK, Martines JC, et al; Lancet Breastfeeding Series Group. Why invest, and what it will take to improve breastfeeding practices? Lancet. 2016; 387: 491-504. https://doi.org/10.1016/S0140-6736(15)01044-2.

12. Zhang Z, Zhu Y, Zhang L, Wan $\mathrm{H}$. What factors influence exclusive breastfeeding based on the theory of planned behaviour. Midwifery. 2018; 62: 177-82. https://doi.org/ 10.1016/j.midw.2018.04.006.

13. Jama NA, Wilford A, Masango Z, Haskins L, Coutsoudis A, Spies L, et al. Enablers and barriers to success among mothers planning to exclusively breastfeed for six months: a qualitative prospective cohort study in KwaZulu-Natal, South Africa. Int Breastfeed J. 2017; 12: 43.

https://doi.org/10.1186/s13006-017-0135-8.

14. Li jing, Qian yan, Liu huijuan, Wei chunlei, Wu juanqin. Analysis of breastfeeding status and influencing factors in Jiaxing City. Zhejiang medicine. 2007; 29:992-94. https://doi.org/10.3969/j.issn.1006-2785.2007.09.045.

15. von Elm E, Altman DG, Egger M, Pocock SJ, Gøtzsche PC, Vandenbroucke JP; STROBE Initiative. The Strengthening the Reporting of Observational Studies in Epidemiology (STROBE) Statement: guidelines for reporting observational studies. Int J Surg. 2014; 12: 1495-9. https://doi.org/10.1016/j.ijsu.2014.07.014.

16. World Health Organization. National implementation of the baby-friendly hospital initiative 2017. ISBN 978-92-4-151238-1. Available from: https://apps.who.int/iris/bitstream/handle/10665/255197/9789241512381-eng.pdf;sequence=1. Accessed 20 October 2021.

17. Hakala $M$, Kaakinen $P$, Kääriäinen $M$, Bloigu R, Hannula L, Elo S. Maternity ward staff perceptions of exclusive breastfeeding in Finnish maternity hospitals: A cross-sectional study. Eur J Midwifery. 2021; 5: 16. https://doi.org/10.18332/ejm/134846.

18. Hawkins SS, Stern AD, Baum CF, Gillman MW. Evaluating the impact of the baby-friendly hospital initiative on breast-feeding rates: A multi-state analysis. Public Health Nutr. 2015; 18:189-97. https://doi.org/10.1017/S1368980014000238.

19. Cinquetti M, Colombari AM, Battisti E, Marchetti P, Piacentini G. The influence of type of delivery, skinto-skin contact and maternal nationality on breastfeeding rates at hospital discharge in a babyfriendly hospital in Italy. Pediatr Med Chir. 2019; 41:17-21. https://doi.org/10.4081/pmc.2019.207.

20. Jaafar SH, Ho JJ, Lee KS. Rooming-in for new mother and infant versus separate care for increasing the duration of breastfeeding. Cochrane Database Syst Rev. 2016; (8): CD 006641. https://doi.org/10.1002/14651858.CD006641. 
21. Mirkovic KR, Perrine CG, Scanlon KS, Grummer-Strawn LM. Maternity leave duration and fulltime/part-time work status are associated with US mothers' ability to meet breastfeeding intentions. J Hum Lact. 2014; 30: 416-9. https://doi.org/10.1177/0890334414543522.

22. Moshi FV, Akyoo EE, Seif SA. Prevalence and predictor of exclusive breastfeeding among mothers of 0 to 6 months infants from pastoralists and hunters' community in Tanzania; A community based cross-sectional study. East Afr Health Res J. 2021; 5: 82-90. https://doi.org/10.24248/eahrj.v5i1.655.

23. Castillo H, Santos IS, Matijasevich A. Maternal pre-pregnancy BMI, gestational weight gain and breastfeeding. Eur J Clin Nutr. 2016; 70: 431-6. https://doi.org/10.1038/ejcn.2015.232.

24. Yang X, Ip WY, Gao LL. Maternal intention to exclusively breast feed among mainland Chinese mothers: A cross-sectional study. Midwifery. 2018; 57: 39-46. https://doi.org/10.1016/j.midw.2017.11.001.

25. Prior E, Santhakumaran S, Gale C, Philipps LH, Modi N, Hyde MJ. Breastfeeding after cesarean delivery: A systematic review and meta-analysis of world literature. Am J Clin Nutr. 2012; 95: 111335. https://doi.org/10.3945/ajcn.111.030254.

26. Li HT, Luo S, Trasande L, Hellerstein S, Kang C, Li JX, et al. Geographic variations and temporal trends in cesarean delivery rates in China, 2008-2014. JAMA. 2017; 317: 69-76. https://doi.org/10.1001/jama.2016.18663.

27. Hector D, King L, Webb K, Heywood P. Factors affecting breastfeeding practices: applying a conceptual framework. N S W Public Health Bull. 2005;16: 52-5. https://doi.org/10.1071/nb05013.

28. Gebrekidan K, Hall H, Plummer V, Fooladi E. Exclusive breastfeeding continuation and associated factors among employed women in North Ethiopia: A cross-sectional study. PLoS One. 2021; 16 : e0252445. https://doi.org/10.1371/journal.pone.0252445. 